\title{
Closed-Loop Control in a Flame and a Dump Combustor
}

\author{
E. Gutmark, T. P. Parr, K. J. Wilson, D. M. Hanson-Parr, and K. C. Schadow
}

A closed-loop control system was constructed to control the lift-off height of a premixed flame at the lean flammability limit. Control authority was obtained by forcing the initial shear layer of the fuel/air mixture, thus generating small scale vortices which improved the flame stability. The optimal forcing was determined in open-loop control tests, which defined the effective range and related it to the Kelvin-Helmholtz instability of the shear layer. An amplitude modulation (AM) controller was developed using the open-loop frequency as a carrier signal. Bode and Nyquist analyses showed that the flame is stable only in a limited range due to convective time lag, related to the convection time of the vortices. In spite of the high noise level of this turbulent combustion system, it was possible to use linear control theory to analyze the time-averaged transfer functions. The application of lead and lag compensations was studied to extend the stability margins. The control system response to changes in operating conditions such as set-point and flow rates were compared to the open-loop control operation and found to be superior, leading to a small extension of the lean flammability limit relative to an open-loop controller.

The same actuation technique investigated in the flame experiments in conjunction with a different control strategy, are used for active combustion control in the dump combustor. Shear-layer excitation was investigated using acoustic drivers. Parameters affecting the efficiency of the various methods for active combustion control were studied, including frequency, amplitude, and phase information. The effectiveness of the acoustic shear-layer excitation on the combustion characteristics was evaluated. A certain attenuation of the unstable combustion oscillations was obtained with the high frequency acoustic excitation.

\section{Closed-Loop Feedback for Combustion Control}

In certain combustor designs with adverse operational conditions (for example, low chamber pressure), combustion is initiated at a certain stand-off distance from the dump or flameholder. The delay of the onset of combustion is due to the presence of large-scale vortical structures in the shear flow and may result in high amplitude pressure oscillations, low combustion efficiency, and narrow flammability margins.

Passive and active control methods, aimed to modify the flow pattern and reduce the coherence of the vortices, were applied to premixed and diffusion flames with favorable results that stabi-

Presented at the 1991 American Control Conference, Boston, MA, June 26-28, 1991. The authors are with the Research Department, Naval Air Warfare Center-Weapons Division, China Lake, CA 93555-6001. The work was supported by the Office of Naval Research. lized the flame and changed the regions of the chemical reaction [1], [2]. These changes obtained by open-loop control were also observed in closed-loop feedback systems for combustion control [3], [4].

A closed-loop active combustion control system requires some feedback signal between the combustion output and the actuators which control the air and fuel flows. Several investigators studied control methods for the suppression of combustion oscillations. Pressure oscillations [5],[6] or light emission fluctuations [3],[7],[8] have been detected by microphones or photodiodes, suitably filtered, delayed, and amplified, and fed back to different types of actuators. The use of radiation to monitor the combustion process was described originally in [9] and [10].

Loudspeakers were used to impose secondary sound waves into the combustor [3],[5],[6],[8]; an oscillating inlet nozzle was studied to increase the energy loss of the system by changing the upstream boundary conditions [4]; and fuel modulation was explored to modify the heat release distribution in the combustor [11].

Active control experiments resulted also in improved combustion characteristics in a $500 \mathrm{~kW}$ combustor by introducing oscillations into the reaction process [12].

This article describes a closed-loop control system used to stabilize the premixed flame, and tests which were aimed to study the effectiveness of a phase-shift acoustical active control system in a relatively high power combustor of $1 \mathrm{MW}$ with a turbulent inlet flow.

\section{Experimental Arrangement}

\section{Flame System}

A propane/air premixed circular flame was studied [1]. The air was supplied through a $290 \mathrm{~mm}$ long circular pipe with a $19 \mathrm{~mm}$ diameter circular orifice exit. The air flow rate varied in the tests from 20 to $100 \mathrm{l} / \mathrm{min}$, yielding exit velocities of 1.65 to $10 \mathrm{~m} / \mathrm{s}$. The corresponding Reynolds number range was 2100 to 12000 , based on the exit diameter, exit air velocity, and the kinematic viscosity of air at room temperature. The initial velocity RMS was $2 \%$. The propane flow rate was $1.91 / \mathrm{min}$.

A schematic description of the control system is given in Fig. 1. The controlled plant consists of three subsystems: acoustics, flow dynamics, and combustion. All the subsystems have links and are interdependent. The output of the plant, i.e., flame standoff, is monitored by sensors and is being fed back and compared with the set-point conditions and subsequently are used as inputs to generate the necessary commands by the controller.

The sensing element in the present system, which is a photodiode, integrates the image of the flame over a predetermined active area to determine the flame location. The 


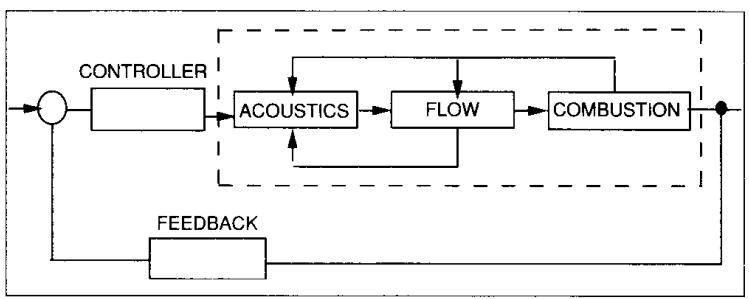

Fig. 1. Block-diagram of the closed-loop combustion control system with interacting subsystems.

photodiode voltage is proportional to the flame lift-off height (i.e., the voltage decreases proportionally to the increased lift-off height). This signal is then compared with the desired height, which is determined by the set point bias. The difference between the two signals is used to modulate the amplitude of the carrier frequency. This frequency is the one determined from open-loop tests and linear stability theory of shear layers to be the most effective in suppressing large-scale vortices and stabilizing the flame. The modulated frequency drives the speaker in the flame cavity via an audio amplifier. The speaker is used as the actuator which controls the flame lift-off distance via changes in the flow structure. The control objective is to maintain the flame lift-off height at a predetermined level. Acoustic modulation at the initial instability frequency of the air/fuel mixture was shown to be capable of varying the lift-off height. The present control system changes the amplitude of the carrier frequency such that a desired flame height is maintained.

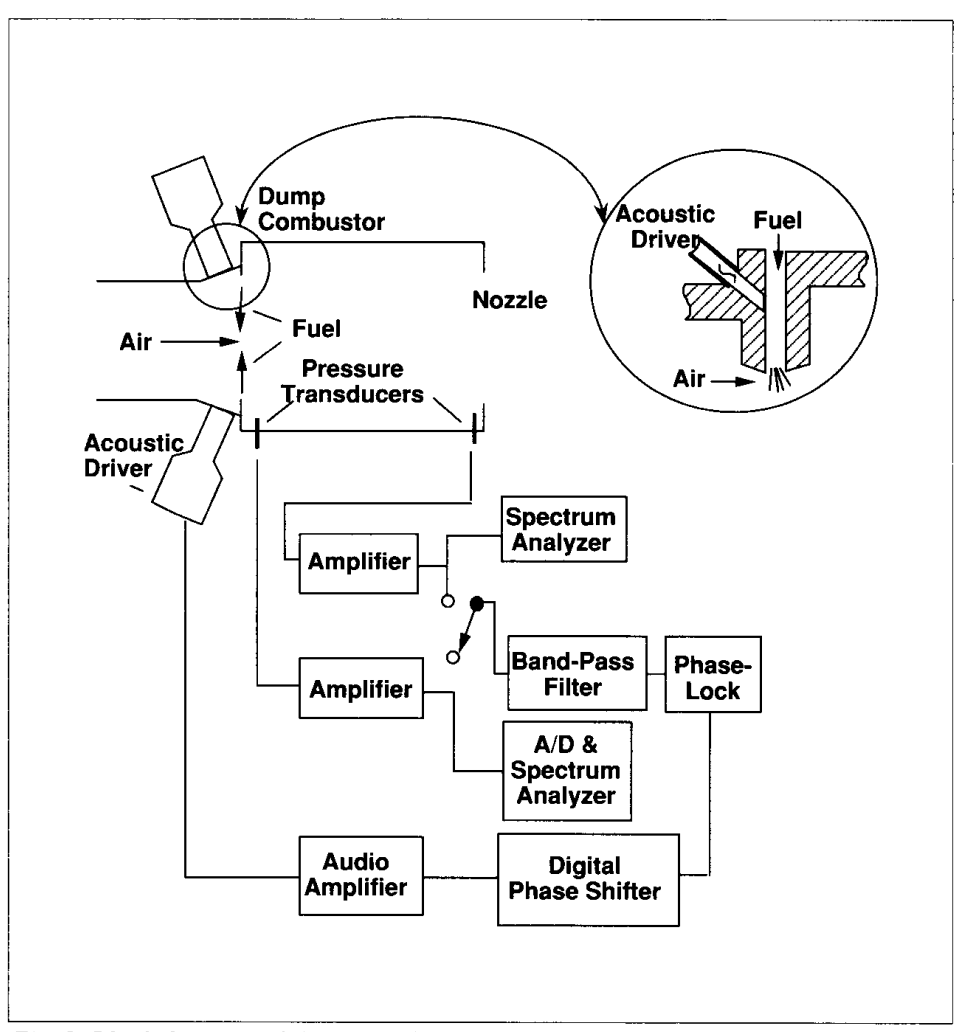

Fig. 2. Block diagram of the closed-loop control system for the dump combustor.

\section{Dump Combustor System}

The schematic of the combustor is shown in Fig. 2. Air is supplied into the combustor through a $D=6.35 \mathrm{~cm}$ diameter circular pipe with a flow straightener $10 \mathrm{D}$ upstream of the dump plane (sudden expansion). The pipe expands through an angle of $7^{\circ}$ to inject the air through an orifice plate of diameter $6.35 \mathrm{~cm}$ into the combustion chamber. The ethylene fuel is injected through four slots in the orifice plate normal to the air flow. Acoustic modulations are superimposed on the fuel stream by four acoustic drivers. Ethylene is injected at a rate of 0.016 to $0.023 \mathrm{lb} / \mathrm{s}$ into 0.255 to $0.484 \mathrm{lb} / \mathrm{s}$ air flow, resulting in an equivalence ratio $\phi$ in the range of 0.71 to 1 . The chamber temperature is 1400 to $1800 \mathrm{~K}$ at ambient pressure.

The steel combustion chamber is circular with a diameter of $2 \mathrm{D}$ and length of $8.4 \mathrm{D}$. The diameter of the exhaust nozzle is $1.65 \mathrm{D}$.

A block diagram of the closed-loop control system is given in Fig. 2. High frequency response pressure transducers, installed near the dump plane (sudden expansion) and near the exhaust nozzle, are used as sensors to monitor the combustion process. Their signals are fed through an amplifier into a band-pass filter which is centered around the most unstable frequency of the combustion pressure oscillations. The filtered signal is used to phase-lock and phase-shift a sinusoidal signal which is fed into four acoustic drivers via audio amplifiers. The acoustic drivers excite acoustic resonance in the connecting tube which modulate the fuel jet and the shear layer at the inlet to the combustion chamber. The shear layer and fuel modulations change the mixing pattern between the air jet and the fuel, and thus can vary the heat release rate.

The dump combustor control system is designed to suppress pressure oscillations in the combustion chamber. These oscillations are excited naturally when the interaction between flow instabilities and the combustion chamber acoustics provide conditions in which pressure oscillations are in phase with the heat release fluctuations (Rayleigh criterion).

\section{Results and Discussion}

\section{Transfer Functions (System Stability)}

Stability analysis of the system was done using the Bode method. The carrier frequency was modulated with bandwidth limited noise and the corresponding response of the sensor was measured. The resulting Bode plot is shown in Fig. 3. The Bode plot is a result of a long time average $(209 \mathrm{~s})$. The system is highly turbulent with high random noise level, yielding a coherence level between the driving signal and the height detector, which was typically lower than 0.3 . Short average Bode plots were extremely noisy, with an RMS fluctuation of about $90^{\circ}$ in angle and about $6 \mathrm{~dB}$ in magnitude, rendering any analysis impossible. However, they show that due to noise the system can occasionally exceed a gain of unity when the phase shift is over $180^{\circ}$, resulting in an unstable controller operation. The quasi 


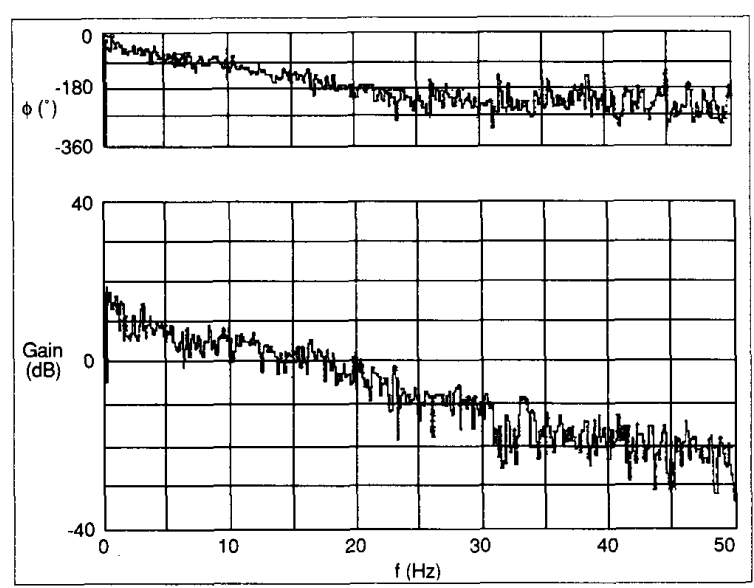

Fig. 3. Averaged Bode plot of the closed-loop control system for the flame. Air flow rate $=38 \mathrm{l} / \mathrm{min}$, fuel flow rate $=1.9 \mathrm{l} / \mathrm{min}$. Flame at the maximum lift-off distance. Averaging time $=209 \mathrm{~s}$.

linear drop of the phase angle with frequency indicates a time lag in the system. The $180^{\circ}$ phase shift occurs over a frequency range of about $18 \mathrm{~Hz}$, which translates to a time lag of $28 \mathrm{~ms}$. Physically, this time lag relates to the convective velocity of the combustible mixture. The acoustic time delay inside the cavity is only of the order of $1 \mathrm{~ms}$. However, when the flame is lifted, the convection time of the vortices from the nozzle to the flame is of the order of $28 \mathrm{~ms}$. To obtain wide stability margins, the gain of the system has to fall below zero decibels before the phase acquires a shift of $180^{\circ}$. In the present system, the gain falls relatively slowly, and considering the noise fluctuations, it still has a positive gain at the phase cross-over point (Fig. 3). This yields a marginally stable system. The spectrum of the $\mathrm{CH} / \mathrm{C}_{2}$ emission signal shows a hump at a frequency of $18 \mathrm{~Hz}$, corresponding to the phase cross-over point. This ability to predict the behavior of the system using linear control theory, in spite of its high noise level, is one of the interesting outcomes of the present work.

The need to reduce the gain at high frequencies, to avoid positive gain at phase shift angles higher than $180^{\circ}$, and the general requirement to reduce high frequency noise, suggest the use of a low-pass filter, which was incorporated in the present system. The improvement of the signal to noise was clear from the coherence plot which showed a significant increase up to 0.7 . However, the rapid roll-off of the gain at a frequency of $16 \mathrm{~Hz}$ is concurrent with an increase in the phase lag, such that $180^{\circ}$ cross-over moves to $8 \mathrm{~Hz}$. For this condition, the flame becomes highly unstable, detaching and reattaching to the flameholder at a nearly steady frequency of $8 \mathrm{~Hz}$.

\section{Response to Changes in Operating Conditions}

The mechanism of controlling the lift-off height is explained here. Previous experiments [1],[2] showed that the flame lift-off phenomenon is related to the existence of large-scale vortices in the air/fuel mixture flow near the nozzle. The large strain rates induced by the vortex roll-up process result in local extinction and the transition of the flame stabilization location to a downstream point. The roll-up pattern of the flow can be altered by acoustically forcing at a frequency which is sufficiently larger than the dominant vortex frequency. This excitation forces the flow to form smaller-scale vortices which in turn shifts the flame stabilization point upstream closer to the nozzle exit. In the present experiment, the flame stand-off distance is monitored by a photodiode and controlled by acoustic drivers. When the flame is higher than desired, the photodiode voltage is less than the set point. The error signal results in increased amplitude of the driving frequency which forces the flame to reduce the lift-off height. When the flame is lower than the set-point, the photodiode voltage is too high, leading to a reduction in the amplitude of the driving frequency and a subsequent increase in the flame lift-off height. The two cases are not symmetric and the difference in the physical system response is discussed in this section. The response of the closed-loop control system to changes in operating conditions was tested by a step change in the set point and in the mixture flow rate.

The step change in the height set-point was first obtained for an open-loop system. The step change in the height set point is manifested in the speaker's driving signal as a sudden change in the modulation amplitude and in the height detector signal as a change in its level. The time response of the flame to the reduction in the forcing amplitude is about $2 \mathrm{~ms}$ while the response to the increase in amplitude is $15 \mathrm{~ms}$. This difference is another manifestation of the convective time delay; the first change occurs when the flame is located near the flameholder, while for the second one the flame is lifted to a larger distance. Another interesting observation is the difference between the rate of change in the two cases. When the flame moves from the low height to the higher, the response rate is only slightly over $1 / 2$ the rate for the reverse motion. The difference is related to the fact that the motion upstream is caused actively by increasing the forcing level, while for the downstream motion the forcing releases the flame to propagate downstream by itself.

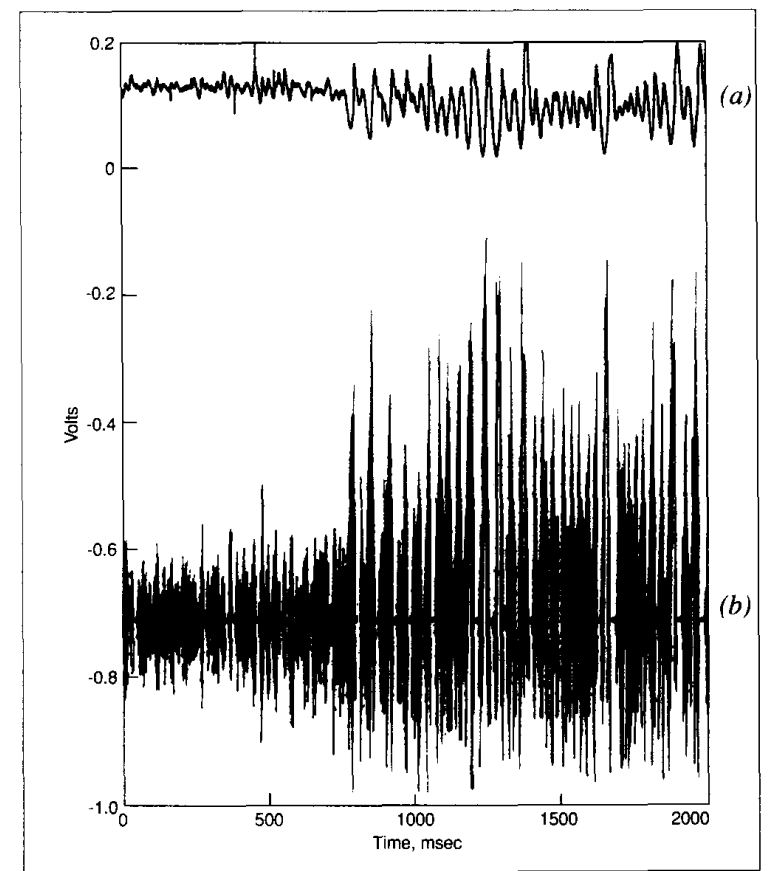

Fig. 4. Closed-loop response to step function change in the air flow rate from 39 to $49 \mathrm{l} / \mathrm{min}$ for flame controller. Fuel flow rate $=1.9$ l/min. (a) Height detector, (b) Control signal. 


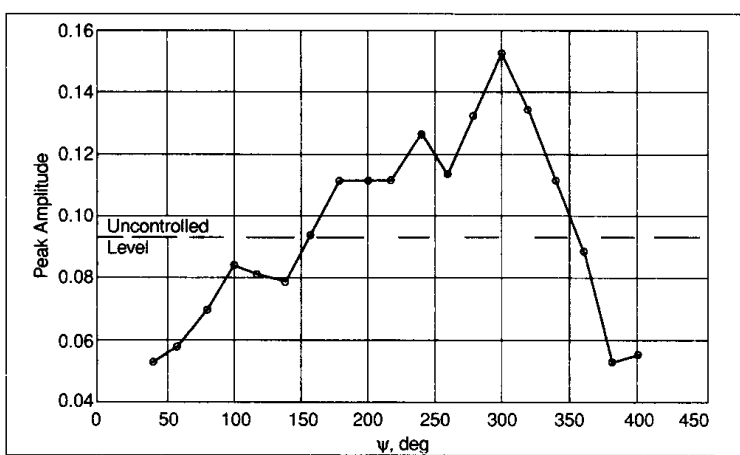

Fig. 5. Variation of the peak amplitude of the combustion instability frequency in a dump combustor with the relative phase shift between the acoustic actuator and the pressure transducer sensing the instability. Pressure sensor and pressure measurements at 1 in downstream of dump.

A similar test was conducted with a closed-loop control. The control system responds to the rapid changes in the set-point by a corresponding change in the modulations. The occasional destabilization of the system was apparent from the oscillations which are superimposed on the sensor and driving signals at a frequency of nearly $20 \mathrm{~Hz}$. The other features of the response are similar to those discussed in relation to the open-loop control

The system response to sudden changes in the fuel/air mixture flow rate were measured in open and closed-loop operations. The open-loop system failed to prevent the blow out of the flame and is not shown here. Fig. 4 shows the closed-loop response to a sudden change in the air flow rate. The air flow rate was increased from 39 to $49 \mathrm{l} / \mathrm{min}$ at $t \approx 800 \mathrm{~ms}$ (both increasing the total flow speed and leaning out the mixture). The open-loop control sys tem could not respond to this change which destabilized the flame. The instability eventually led to extinction for $t>1600$ ms. The closed-loop system responded to the same change by increasing the amplitude of the speaker's driving signal. The flame was sustained at a flow rate at which it was blown out with an open-loop controller, at the desired height, though with increased fluctuations.

\section{Control of a Dump Combustor at Low Air Flow Rate}

Pressure oscillations in a dump combustor are caused by interaction between flow instabilities and acoustic resonance modes of the combustion chamber. When the acoustic resonant frequency matches the flow's unstable range of frequencies, the pressure perturbations induce velocity fluctuations in the thin shear layer at the inlet, which subsequently grow and cause roll-up of the air and fuel mixture into vortices. This roll-up enhances small-scale mixing and consequently the combustion process and therefore the heat release becomes periodic. When the periodic heat release is in phase with the pressure oscillations, the positive feedback destabilized the combustion and the pressure oscillations get amplified leading to unstable operation of the combustor.

Suppression of instability by active control relies on two principles, one is to decouple the heat release from the pressure oscillations and the other is to reduce the coherence of the vortices formed at the nozzle
Closed-loop control of the pressure oscillations in the dump combustor was employed by varying the phase of the acoustic driving signal relative to the pressure fluctuations measured 1 inch downstream of the dump plane. The speakers were driven in a range of $\psi=40^{\circ}$ to $400^{\circ}$ phase shift angles relative to the pressure signal. The variation of the peak amplitude of the instability frequency as a function of the phase shift angle is shown in Fig. 5. A reduction of $47 \%$ in the peak amplitude relative to the uncontrolled combustion was obtained at $\psi=40^{\circ}$. The horizontal dashed line on the graph represents the amplitude of the uncontrolled combustion. In a range of phase shift angles between $\psi=170^{\circ}$ to $350^{\circ}$, the amplitude of oscillations was increased relative to the unforced combustion, with a peak increase of $50 \%$ relative to the unforced conditions at $\psi=300^{\circ}$.

Concurrent with the changes of amplitude, there is a cyclic variation of the instability frequency. A minimum frequency of $280 \mathrm{~Hz}$ was measured at the highest suppression phase angle, while $f=310 \mathrm{~Hz}$ at the point of maximum amplitude. This frequency change did not have a considerable effect on the close-loop performance since the variation range was small relative to the window width of the band-pass filter.

\section{Effect of Control on the Flammability Limit}

The effect of control on the lean flammability limit was studied at two phase shift angles: one corresponding to effective suppression conditions and one at which the instability is augmented. RMS of the pressure oscillations in the combustion chamber were measured as the equivalence ratio (fuel to air ratio) was varied (Fig. 6). The instability of the uncontrolled combustion became highly amplified at $\phi=0.93$. The amplitude of the fluctuations increased and the frequency dropped to near $200 \mathrm{~Hz}$. The high level oscillations continued until the lean flammability limit (extinguished combustion) was reached at $\phi=0.72$. When the flame was controlled at a phase shift angle of $\psi=80^{\circ}$ corresponding to a maximum suppression of the pressure oscillations, the onset of the high amplitude instability was delayed to $\phi=0.81$ and was blown out only at $\phi=0.54$. The onset of large amplitude pressure oscillations was delayed (to $\phi=0.87$ ) also with a phase shift angle of $320^{\circ}$ corresponding to augmentation of the instability, but the flammability limit shifted to a higher $\phi$ of 0.78 .

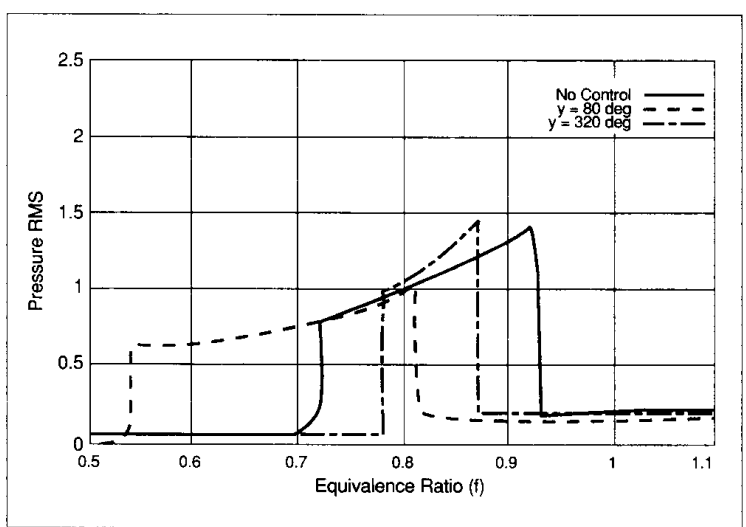

Fig. 6. Comparison of combustion behavior near the lean flammability limit between unforced and controlled combustion, with efficient suppression and instability augmentation. 


\section{Control of the Dump Combustor at High Air Flow Rate}

As the air flow rate increases, the pattern of instability becomes bi-modal and therefore more difficult to control. The unstable frequencies are $310 \mathrm{~Hz}$ and $360 \mathrm{~Hz}$. During a cycle of changed phase in the control measurements the amplitudes of the two peaks change and they become alternately dominant.

Due to this bi-modal spectrum, the window of the band-pass filter was widened to include the two frequencies. However, the switching of the dominant frequencies complicates the locking of the driving signal and causes shifts in the relative phase due to the variation of the phase caused by the band pass filter when operating off the center frequency. The amplitude of the $310 \mathrm{~Hz}$ oscillations shows cyclic response to the forcing, with minimum values at $300^{\circ}$ and a maximum at $180^{\circ}$. The reduction at $310^{\circ}$ is more than $35 \%$ relative to the unforced amplitude. The higher frequency of $360 \mathrm{~Hz}$ showed lower variations relative to the unforced level with a minimum at $180^{\circ}$ and a maximum at $360^{\circ}$. The maximum reduction of amplitude was only $20 \%$.

The effect of control on the lean flammability limit was tested also for the high flow rate. The unforced combustion was compared with a controlled condition at a phase shift of $\psi=340^{\circ}$. The effect was very minor with a slight increase of the equivalence ratio which corresponds to the onset of high-level instability, from $\phi=0.78$ to 0.81 .

\section{Flame Control}

The premixed flame exhibits a turbulent nature at the lean flammability limit especially for high fuel flow rates, which require high air flow rate and high Reynolds number at this condition. Analysis of the dynamic frequency-response of the system showed a highly nonlinear system with large noise level. The nonlinearity of the systems is primarily related to nonlinear flow processes in the shear layer such as vortex roll-up and merging. The nonlinearity of the system was bypassed by introducing the amplitude-modulation (AM) control scheme. Openloop control tests and linear stability theory of shear layers were used to select an effective single frequency for the control signal. By forcing the shear layer at this most amplified frequency, the merging process is suppressed such that the mechanism for generating vortices at the subharmonic frequencies is eliminated. The forcing is also supplying effectively the required control authority, as demonstrated in open-loop control tests. The response of the lifted flame to this forcing is linear in a certain range, i.e., increased amplitude yields a corresponding linear reduction of the lift-off distance. The linear behavior is restricted at the two extremes: on the lower side by the flameholder and at the downstream side by the blow-out limit.

The AM controller did not solve the noise problem; however, surprisingly, the information of long time-averaged Bode or Nyquist plots was sufficient to obtain a good prediction of the system stability margins based on linear control theory.

The stability margins of the system were limited by a convective time lag inherent to the plant due to the finite time which is required for the vortices to be convected from the flameholder to the flame. Attempts to reduce this problem by adding a lead compensation network are hampered by the fact that the improvements in the phase angle is accompanied by an increase in gain at high frequencies which causes reduced stability margins. The time lag problem is alleviated by operating the flame at the utmost upstream location, thus reducing the convective time and reducing the fluctuations level.

Further improvement of the system is planned by applying nonlinear control at the boundaries utilizing a rate feedback controller outside the linear range.

\section{Dump Combustor Control}

Active combustion control was tested in a dump combustor for two air mass flow rates corresponding to 33 and $66 \mathrm{~m} / \mathrm{s}$ at the inlet. In both low and high air flow rates the fuel modulation suppressed the instability at a certain range of phase shift angles and increased the instability level at other angles. For the low flow rate, a $47 \%$ suppression was obtained at a phase angle of $40^{\circ}$ while an augmentation of $50 \%$ was measured at $300^{\circ}$. The total RMS was reduced by $12 \%$ and increased by $8 \%$ at the same frequencies, respectively. The reduced oscillations produced also a delayed onset of high level instability oscillations at the lean flammability limit and an extension of this limit from an equivalence ratio of 0.72 to 0.54 .

Increasing the amplitude of the acoustic forcing resulted in a higher suppression of the peak amplitude at the effective phase shift angles, which was nearly proportional to the forcing level up to a certain limit of forcing level where leveling off occurred. The total RMS reduction was less affected by the forcing level.

High flow rate combustion instability was characterized by a bi-modal spectrum which reduces the effectiveness of the phaselock control due to the varying dominant frequency. It is required for such operational conditions to widen the window of the band-pass filter which subsequently introduces phase errors due to the dependence of the phase on the frequency when the dominant frequency is off the center frequency of the filter. The effectiveness of the control system is thus reduced, such that the highest suppression was $35 \%$, at a phase shift angle of $300^{\circ}$, while the highest increase was $20 \%$ at $180^{\circ}$. At this flow rate the control did not have any effect on the combustion instability and blow-out at the lean flammability limit. In order to control a bi-modal instability, a dual-loop controller is preferred. The two unstable components can be suppressed independently by optimizing the time delay for each one of them. Such system was shown to be more effective in the simultaneous suppression of the two modes and extension of the lean flammability limit.

\section{Acknowledgment}

The authors would like to thank Dr. G. Hewer, D. Klabunde, and D. Philbrick for discussions on the control aspects of the work.

\section{References}

[1] E. Gutmark, T. P. Parr, D. M. Parr, and K. C. Schadow, "Stabilization of combustion by controlling the turbulent shear flow structure," presented at 7th Symp. Turbulent Shear Flows, Stanford, CA, Aug. 1989.

[2] E. Gutmark, T. P. Parr, D. M. Hanson-Parr, and K. C. Schadow, "On the role of large and small scale structures in combustion control," Combustion Sci. Technol., vol. 66, pp. 107-126, 1989.

[3] T. Poinsot, F. Bourienne, S. Candel, and E. Esposito, "Suppression of combustion instabilities by active control," J. Propul. \& Power, vol. 5, pp. 14-20, Jan-Feb. 1989

[4] G. J. Bloxsidge, A. P. Dowling, N. Hooper, and P. J. Langhorne, "Active control of reheat buzz," AIAA J., vol. 26, pp. 783-790, July 1988.

[5] A. Gulati and R. Mani, "Active control of unsteady combustion induced oscillations," presented at 28th Aerospace Sci. Meet., AIAA Pap. 90-0270, Jan. 1990. 
[6] W. Lang, T. Poinsot, and S. Candel, "Active control combustion instability," Combustion and Flame, vol. 70, pp. 281-289, 1987.

[7] K. R. McManus, V. Vansburger, and C. T. Bowman, "Combustor performance enhancement through direct shear layer excitation," Combustion and Flame, vol. 82, pp. 75-92, 1990.

[8] E. Gutmark, T. P. Parr, D. M. Hanson-Parr, and K. C. Schadow, "Use of chemiluminescence and neural networks in active combustion control," presented at 23rd Int. Symp. Combustion, pp. 1101-1106, 1990.

[9] R. R. John and M. Summerfield, "Effect of turbulence on radiation intensity from propane-air flames," Jet Propul., vol. 27, p. 169, 1957.

[10] J. Diederichsen and R. D. Gould, "Combustion instability: Radiation from premixed flames of variable burning velocity," Combustion and Flame vol. 9, pp. 25-31, 1965 .

[11] P. J. Langhorne, A. P. Dowling, and N. Hooper, "Practical active control system for combustion oscillations," J. Propulsion and Power, vol. 6, no. 3, pp. 324-332, 1990.

[12] K. C. Schadow, E. Gutmark, and K. J. Wilson, "Active combustion control in a coaxial dump combustor," Combustion Sci. Technol., vol. 81, pp. $285-300,1992$.

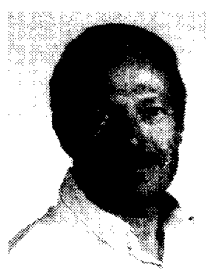

Ephraim (Effie) Gutmark has been with the Propulsion Research Branch of the Engineering Sciences Division since 1986. He has been working in the areas of air breathing propulsion, interaction of combustion and fluid mechanics, passive and active control of combustion in subsonic and supersonic flows and aeroacoustics. His work has been reported in over 160 publications and technical reports. He was a Visiting Professor at the University of Southern California where he performed research in the area of turbulent shear layers, boundary layers, and heat transfer. He received the B.S. (1969), M.S. (1971), and Ph.D. (1976) degrees in aerospace and aeronautical engineering from the Technion, Israel Institute of Technology.

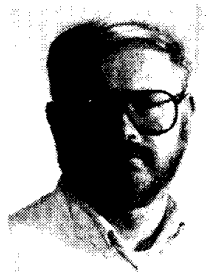

Timothy P. Parr has been at the Naval Air Warfare Center Weapons Division since 1981, working in the areas of advanced laser diagnostics, with applications to energetic materials combustion chemistry and kinetics, turbulent combustion including 3D imaging of turbulent flames, plume diagnostics, and active combustion control. He has more than 120 publications in the fields of solid propellant and propulsion research. He obtained the B.S. degree in chemistry in 1971 and the $\mathrm{Ph} . \mathrm{D}$ degree in chemical physics (molecular beam reaction dynamics) in 1976 from the University of California at Berkeley, and did post-doctoral work at the University of California at Santa Barbara (1976-1980), in the area of meta-stable atom reaction dynamics.

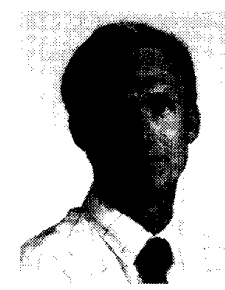

Kenneth J. Wilson is a member of the Propulsion Research Branch of the Engineering Sciences Division. For the past 12 years, he conducted research in the areas of ramjet propulsion systems, supersonic mixing, and active combustion control. His work is published in open literature and technical reports. He received the bachelor's degree in aerospace engineering from lowa State University in 1980.

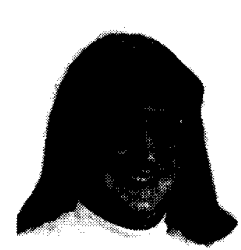

Donna Hanson-Parr has been at the Naval Air Warfare Center Weapons Division since 1981, working in the areas of advanced laser and optical diagnostics with applications to energetic materials combustion chemistry and kinetics, turbulent combustion, and plume diagnostics. She has also made use of fractal theory and 3D imaging in analysis of complex fluid dynamic data, and has over 100 publications in the fields of solid propellant and propulsion research. She obtained the B.S. degree in chemistry and mathematics from Marquette University, Milwaukee, WI, in 1976, and the Ph.D. degree in experimental physical chemistry (meta-stable atom reaction dynamics) from the University of California at Santa Barbara in 1980

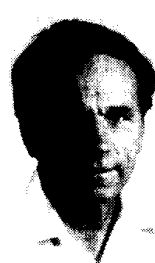

Klaus C. Schadow is the Head of the Propulsion Research Branch of the Engineering Sciences Division, Research Department. He joined the Naval Weapons Center in 1970 . He performs active and passive shear-flow control research in nonreacting and combustion experiments in subsonic and supersonic combustion systems. His research has been published through open literature and technical reports and has been presented at technical meetings. From 1962 to 1970 , he was employed at the DFVLR in Germany, working on spray combustion, thrust vector control, and boron combustion. Dr. Schadow received his Diploma in Mechanical Engineering from the Technical University in Hannover (1962), and the $\mathrm{Ph} . \mathrm{D}$. degree in general engineering from the Technical University in Munich (1975) 